\title{
Grupo motivacional en unidad hospitalaria desintoxicación, su influencia en mantenimiento de la abstinencia y retención al tratamiento tras alta
}

\section{Motivational interviewing group at inpatient detoxification, its influence in maintaining abstinence and treatment retention after discharge}

\author{
Diana Bachiller*,******, Lara Grau-López*,******, Carmen Barral*,**,***, Constanza Daigre*, \\ **, Cristina Alberich**, Laia Rodríguez-Cintas*,**, Sergi Valero**, Miquel Casas**,***, Carlos \\ RONCERO*,**,*** \\ * CAS Vall d’Hebron. Servicio de Psiquiatría. Hospital Universitario Vall d’Hebron. - ASPB. Barcelona, España. \\ ** Servicio de Psiquiatría. Hospital Universitario Vall d'Hebron, Barcelona, España - CIBERSAM \\ *** Departamento de Psiquiatría. Universidad Autónoma de Barcelona, España.
}

\section{Resumen}

La tasa de recaída en el consumo al alta de una Unidad de Desintoxicación Hospitalaria es elevada. El objetivo de este estudio piloto es valorar los factores sociodemográficos, clínicos y terapéuticos asociados al mantenimiento de la abstinencia de pacientes que han participado en un grupo psicoterapéutico breve de corte motivacional durante su ingreso para la desintoxicación.

Un total de 46 pacientes con diagnóstico de trastorno por dependencia a sustancias, según DSM-IV, ingresados en Unidad de Desintoxicación Hospitalaria participaron en un grupo breve de corte motivacional. Se midieron variables sociodemográficas y clínicas, así como la motivación al cambio (University of Rhode Island Change Assessment, URICA) y la satisfacción con el tratamiento grupal (Cuestionario de Percepciones de Tratamiento, CPT). El mantenimiento de la abstinencia y la retención al tratamiento, dos meses tras el alta, se evaluaron mediante llamadas telefónicas semanales. Se realizó un análisis de supervivencia. Los resultados muestran que ser varón, tener cogniciones del estadio de mantenimiento del cambio al alta y tener una buena satisfacción con la terapia grupal y su terapeuta durante el ingreso, se asocia con mayor tiempo en abstinencia tras el alta.

El abordaje psicoterapéutico grupal breve de corte motivacional, en pacientes ingresados para la desintoxicación, se relaciona con mayor probabilidad de mantenimiento de abstinencia y de retención al tratamiento posterior.

Palabras clave: Entrevista Motivacional, trastorno por uso de sustancias, recaída, desintoxicación hospitalaria, terapia grupal.

\begin{abstract}
The relapse rate after discharge from inpatient detoxification is high. The objective of this pilot study is to assess the sociodemographic, clinical and therapeutic factors associated with maintaining abstinence in patients who participated in a brief motivational interviewing group during admission for detoxification.

A total of 46 patients, diagnosed substance dependent according to DSM -IV, and admitted to the Hospital Detoxification Unit, participated in a brief motivational interviewing group. Sociodemographic, clinical, motivation to change (University of Rhode Island Change Assessment, URICA) and satisfaction with the treatment group (Treatment Perceptions Questionnaire, CPT) data were collected. Abstinence and treatment retention two months after discharge were assessed by weekly telephone calls. A survival analysis was performed. Being male, having more cognitions of the maintenance stage of change at discharge, being satisfied with group therapy and therapist during hospitalization are associated with longer abstinence after discharge. The brief motivational interviewing group approach with patients admitted for detoxification is related to greater likelihood of maintaining abstinence and subsequent treatment retention.

Key words: Motivational Interviewing, substance-related disorders, relapse, inpatient detoxification, group therapy.
\end{abstract}




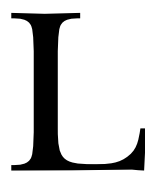

a adicción es un trastorno psicológico crónico y recurrente. El porcentaje de recaída tras un ingreso hospitalario de desintoxicación es muy alto (Santa Ana, Wulfert y Nietert, 2007). A los seis meses tras el alta, permanecen abstinentes a distintas sustancias entre el $12,5 \%$ y el $27,8 \%$ de los ingresados, aumentando en un $32 \%$ en el caso de la cocaína (Grau-López et al., 2012; Shaw, Waller, Latham, Dunn y Thomson, 1998), mientras que sólo el 17,4\% permanece sin consumir alcohol al año (John, Veltrup, Driessen, Wetterling y Dilling, 2003).

Algunas variables sociodemográficas y clínicas se han relacionado con la recaída, como son el género, la convivencia, el nivel educativo, la situación laboral, los antecedentes legales, los antecedentes familiares de adicción a sustancias, la comorbilidad con otro trastorno mental (diagnóstico dual), el tipo de sustancia adictiva, los años de evolución de la adicción, el policonsumo, los ingresos previos o realizar un consumo, en forma de atracón, a modo de despedida. Dichas variables pueden ser indicadoras de la gravedad de la adicción (Arias et al., 2013; García Rodríguez et al., 2005; Goeb, Coste, Bigot y Ferrand, 2000; Grau-López et al., 2012; John et al., 2003; Márquez-Arrico y Adán, 2013; Schellekens, de Jong, Buitelaar y Verkes, 2014).

La escasa motivación al cambio es otro factor importante que se ha relacionado con la recaída al alta (Loeber, Kiefer, Wagner, Mann y Croissant, 2009). En este área, la entrevista motivacional, desarrollada por Miller y Rollnick (2002), centra el tratamiento de las adicciones en aumentar la motivación interna del paciente al cambio. La intervención, con este enfoque terapéutico, se ha relacionado con una reducción del uso de sustancias adictivas, un efecto positivo sobre los procesos de cambio y un aumento de la retención en tratamiento (Wagner y Ingersoll, 2012). También se ha demostrado la eficacia de la entrevista motivacional en formato breve, sin embargo, dicha efectividad depende de variables del paciente asociadas a la severidad de la adicción, sobre las que se necesita realizar más estudios (Vasilaki, Hosier y Cox, 2006).

La retención al tratamiento es un factor importante en la prevención de recaídas, por lo que, si se incorporan estrategias para mejorar la retención, se mejora el pronóstico (NIDA, 2010). Sin embargo, la falta de retención al tratamiento es muy común en los pacientes con adicciones (Chutuape, Katz y Stitzer, 2001), siendo superior a otro tipo de trastornos mentales (Wierzbicki y Pekarik, 1993). Las tasas de inicio de tratamiento de deshabituación posterior a un ingreso mejoran con la introducción de grupos durante el ingreso, e información de grupos al alta (Frydrych, Greene, Blondell y Purdy, 2009).

Por otra parte, se ha propuesto que la satisfacción con el tratamiento psicológico, recibido durante el ingreso, es otro factor importante en la retención y en la abstinencia posterior, aunque existen escasos estudios al respecto (Ino, Saka, Yamashiro, Cho y Torituka, 2006).
Por lo tanto, la desintoxicación hospitalaria no debe limitarse al manejo farmacológico, sino que debería promover intervenciones psicoterapéuticas que fomenten el mantenimiento de la abstinencia y la vinculación al tratamiento posterior (Driessen, Veltrup, Junghanns, Przywara y Dilling, 1999; Myrick, Anton y Kasser, 2003, O’Farrell, Murphy, Alter y Fals-Stewart, 2008). Sin embargo, existe escasa investigación relativa a la introducción de grupos terapéuticos en las unidades de desintoxicación (Berman, Forsberg, Durbeej, Kallmen y Hermansson, 2010; John et al., 2003; Loeber et al., 2009; Santa Ana et al., 2007; Schilling, El-Bassel, Finch, Roman y Hanson., 2002; Stetter, Zahres, Batra y Mann, 1995).

Por dicho motivo, el objetivo de este estudio piloto es describir qué factores sociodemográficos, clínicos y terapéuticos se asocian al mantenimiento de la abstinencia, en pacientes que participan en un grupo de corte motivacional durante el ingreso de desintoxicación, a los dos meses de seguimiento. La hipótesis del estudio es que existen diferencias en variables clínicas y sociodemográficas asociadas a la abstinencia. Respecto a las variables terapéuticas, la hipótesis es que a mayor motivación por el cambio al alta y mayor satisfacción con la terapia grupal, mayor duración de la abstinencia y mayor probabilidad de mantener la retención en un tratamiento posterior.

\section{Método}

\section{Diseño del estudio}

Se realizó un estudio descriptivo longitudinal, de dos meses de seguimiento, con 46 pacientes.

\section{Participantes}

De los 58 pacientes ingresados en la Unidad Hospitalaria de Desintoxicación (UHD) del Hospital Universitario Vall d'Hebron, desde Mayo a Septiembre del 2012, diagnosticados de trastorno de dependencia a sustancias, según criterios DSM-IV-TR, se incluyeron 46 sujetos en el estudio, los cuales participaron voluntariamente en el grupo terapéutico y aceptaron realizar seguimiento durante dos meses tras el alta hospitalaria (Figura 1). Los pacientes firmaron el consentimiento informado del estudio, aprobado por el comité ético del hospital.

Se excluyeron aquellos pacientes que no finalizaron el ingreso hospitalario, los que participaron en otros protocolos de investigación (ensayos clínicos), y los que rechazaron participar en el grupo terapéutico o en el estudio. De los 46 sujetos incluidos en el estudio, 43 finalizaron el período de seguimiento.

Los pacientes no recibieron remuneración alguna por los datos aportados al estudio. 


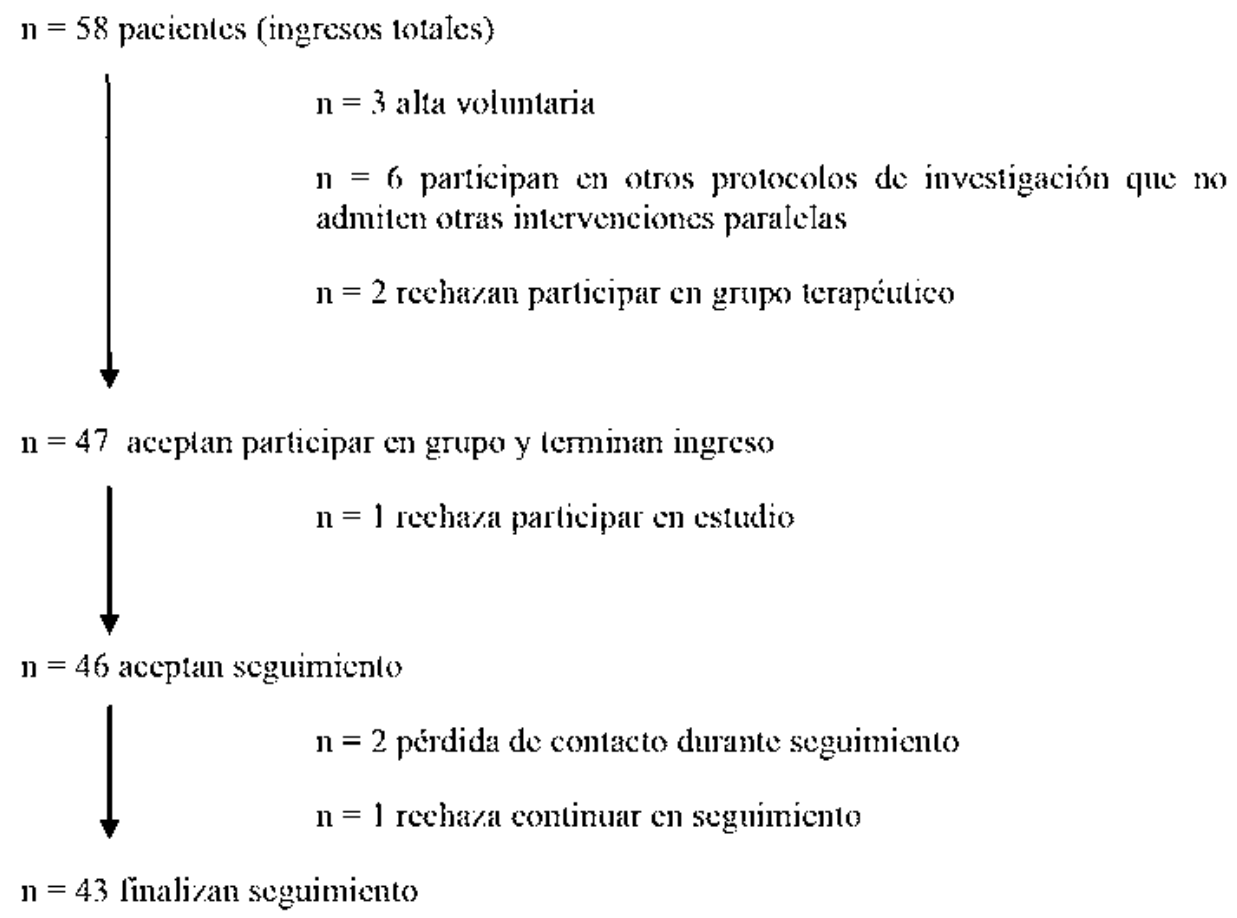

Figura 1. Flujo de la muestra de estudio

\section{Instrumentos}

\section{Medidas pregrupo terapéutico.}

Registro diseñado adhoc. En el que se recogieron datos acerca de variables sociodemográficas (género, edad, nacionalidad, convivencia, estudios, situación laboral, antecedentes legales) y clínicas (antecedentes familiares de adicción a sustancias, número de sustancias con abuso o dependencia actual, tipo de sustancia principal motivo de ingreso, años de evolución de la dependencia a dicha sustancia, ingresos previos, bien sea por la dependencia o por desestabilización psicopatológica, consumo de la sustancia en forma de atracón a modo de despedida previo al ingreso).

Para el registro de la existencia o no de diagnóstico dual se utilizaron los siguientes instrumentos:

- SCID-I. Entrevista clínica estructurada para los trastornos del eje I del DSM-IV, en su adaptación española (First, Spitzer, Gibbon y Williams, 1999).

- SCID-II. Entrevista clínica estructurada para los trastornos de la personalidad del eje II del DSM-IV, en su adaptación española (First, Gibbon, Spitzer, Williams y Benjamin, 1999).

\section{Medidas postgrupo terapéutico.}

Escala URICA. Escala de evaluación para el cambio de la Universidad de Rhode Island (McConnaughy, Prochaska y Velicer, 1983); versión española de Rojas y Espinoza (2008). Este instrumento evalúa en qué medida el sujeto presenta comportamientos o cogniciones propias de cada estadio de cambio, según la Teoría del Cambio de Prochaska y DiClemente (1982). Mide 4 subescalas: precontemplación, contemplación, acción y mantenimiento. A mayor puntuación, más cogniciones propias de cada estadio. La puntuación Readiness to Change (RTC) se obtiene a partir de las 4 anteriores, y mide la disposición global del individuo al cambio. La escala cuenta con una consistencia interna aceptable 0.69-0.89 (McConnaughy et al., 1983; McConnaughy, DiClemente, Prochaska y Velicer, 1989).

CPT. Cuestionario de Percepciones de Tratamiento (Marsden et al., 1998), versión española (Mandersen et al., 2001). Es un cuestionario breve, con puntuaciones comprendidas entre 0 y 40 . Está formado por dos factores, con puntuaciones entre 0 y 20: las percepciones sobre los terapeutas y las percepciones sobre la terapia. A mayor puntuación, mayor satisfacción con el tratamiento. Este instrumento cuenta con un coeficiente aceptable de correlación interclase de 0,57 en muestra española (Mandersen et al., 2001).

\section{Medidas de seguimiento.}

Información autorreportada sobre la abstinencia y la retención al tratamiento de deshabituación a sustancias, a través de seguimiento telefónico semanal de dos meses de duración. Se utilizaron los siguientes criterios para cada variable:

- Abstinencia de la sustancia principal motivo de ingreso. Respecto al consumo del alcohol, se estableció como fin de la abstinencia el beber durante cinco días seguidos, 
o consumir más de cinco unidades del alcohol en un día (Volpicelli et al., 1997). Para el resto de sustancias, se consideró el fin de la abstinencia cuando el paciente admite tres consumos en una semana, o un patrón similar al anterior al ingreso (Grau-López et al., 2012).

- Retención en el tratamiento posterior. Haber acudido, o tener cita concertada, en, al menos, seis de las ocho semanas en las que se realizó el seguimiento telefónico.

\section{Procedimiento}

Durante su ingreso en la UHD, los pacientes siguieron tratamiento farmacológico de desintoxicación. El psiquiatra instauró pautas descendentes de benzodiacepinas hasta su retirada para la desintoxicación de cada sustancia, así como fármacos coadyuvantes para tratar los síntomas comórbidos. Asímismo, en algunos casos se prescribieron fármacos específicos para la adicción como antabús o metadona.

Se informó sobre la naturaleza del estudio y se les propuso participar en el grupo de terapia breve. La no entrada en el estudio no impidió su participación en el grupo.

El tratamiento psicoterapéutico que se realizó tiene como base la entrevista motivacional (Miller et al., 2002; Wagner et al., 2012). Los objetivos terapéuticos principales fueron dos: aumentar la motivación para el mantenimiento de la abstinencia tras el alta, y motivar la retención al tratamiento posterior. La duración de cada sesión fue de 45 minutos, y la frecuencia, tres veces por semana. El tamaño grupal osciló en un rango entre cuatro y cinco participantes. Los grupos fueron abiertos, con sesiones independientes entre sí no protocolarizadas, para facilitar la constante incorporación y salida de miembros al grupo, y adaptarse al contexto de la unidad. Si el terapeuta valoraba que el estado de alguno de los participantes no era el adecuado, debido a la presencia de síntomas de intoxicación, abstinenciales, efectos sedativos producidos por los fármacos o consecuencias adversas derivadas de cualquier enfermedad física, no participaban en dicha sesión. El papel de los conductores del grupo (psicóloga clínica en coterapia con un psicólogo interno residente) fue el de dinamizar los temas que planteaba el propio grupo de participantes. Los psicoterapeutas cuentan con formación estándar en entrevista motivacional y con amplia experiencia en terapia grupal con drogodependientes.

El ingreso en la UHD de la muestra tuvo una duración media de 12.2 días $(D T=5.24$; rango 5 - 36). El número de sesiones media del tratamiento grupal motivacional fue de $4.07(D T=2.45$; rango 2 - 16) .

Se solicitó la autorización a los participantes del estudio para la evaluación previa, posterior al grupo, y durante dos meses tras el alta. Al alta hospitalaria, todos recibieron una cita de seguimiento en su centro de referencia. La evaluación durante el seguimiento consistió en contactar telefónicamente, cada semana, durante dos meses tras el alta, para evaluar si mantenían la abstinencia a la sustancia principal y si continuaban en tratamiento.

\section{Análisis estadístico}

En primer lugar, se describen las variables sociodemográficas y clínicas de la muestra. Para el análisis de la relación entre las variables sociodemográficas, clínicas y terapéuticas con la abstinencia tras el alta, se realiza la comparación de medias mediante la prueba U de Mann-Whitney para variables continuas. En el caso de variables categóricas, se utiliza la prueba Chi Cuadrado, excepto cuando al menos una casilla tenga una frecuencia esperada inferior a 5 , donde se aplica la corrección de Fisher. Se pone a prueba, mediante correlación de Pearson, la posible asociación entre las variables resultantes de dicho análisis antes de incluirlas en la regresión logística, y se escoge aquella con mayor tamaño del efecto, medido por la d de Cohen. Se realiza una regresión logística, con el método de introducción de variables hacia delante, para determinar si las variables que se asocian con la recaída lo hacen de manera independiente. Por último se emplea el modelo de Cox, método adelante, razón de verosimilitud, para determinar la capacidad predictiva de dichos factores en la duración de la abstinencia. Se usa Kaplan-Meier para la función de supervivencia. Los datos se analizaron con el paquete estadístico SPSS versión 20.0.

\section{Resultados}

Se describen las características sociodemográficas y clínicas de los sujetos que participan en el estudio (Tabla 1). La edad media es de 44.9 años $(D T=10.60$; rango 25 - 68).

Tabla1

Descripción de la muestra ( $n=46)$. Variables sociodemográficas y clínicas

\begin{tabular}{|c|c|}
\hline \multicolumn{2}{|c|}{ Variables sociodemográficas } \\
\hline \multicolumn{2}{|l|}{ Género } \\
\hline Varón & $58.7 \%$ \\
\hline Mujer & $41.3 \%$ \\
\hline \multicolumn{2}{|l|}{ Convivencia } \\
\hline Solo & $17.4 \%$ \\
\hline Con familia & $82.6 \%$ \\
\hline \multicolumn{2}{|l|}{ Estudios } \\
\hline Básicos & $69.6 \%$ \\
\hline Superiores & $30.4 \%$ \\
\hline \multicolumn{2}{|l|}{ Trabajo } \\
\hline Activo & $8.7 \%$ \\
\hline No activo & $91.3 \%$ \\
\hline Antecedentes Legales (sí) & $17.4 \%$ \\
\hline \multicolumn{2}{|l|}{ Variables Clínicas } \\
\hline Antecedentes Familiares Tóxicos (sí) & $73.9 \%$ \\
\hline Patología Dual (sí) & $71.7 \%$ \\
\hline \multicolumn{2}{|l|}{$\mathrm{N}^{0}$ sustancias dependencia actual } \\
\hline 1 & $52.2 \%$ \\
\hline 2 ó más de 2 (politoxicomanía) & $47.8 \%$ \\
\hline \multicolumn{2}{|l|}{ Tipo sustancia principal } \\
\hline Depresora & $78.3 \%$ \\
\hline Estimulante & $21.7 \%$ \\
\hline Años evolución dependencia sustancia principal & $\begin{array}{l}(21.26,10.45) \\
\text { (rango, 3-50) }\end{array}$ \\
\hline Ingresos Previos (sí) & $67.4 \%$ \\
\hline Despedida previa al ingreso (sí) & $50 \%$ \\
\hline
\end{tabular}

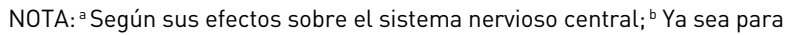
desintoxicación o por desestabilización psicopatológica 
Tabla 2

Descripción de las variables asociadas con la abstinencia a dos meses tras el alta (n=43)

\begin{tabular}{|c|c|c|c|c|}
\hline \multicolumn{5}{|c|}{ Variables sociodemográficas y clínicas } \\
\hline & ABSTINENCIA $n=22$ & NO ABSTINENCIA $n=21$ & Test & $\mathrm{p}$ \\
\hline \multicolumn{5}{|l|}{ Género } \\
\hline Varón & $77.3 \%$ & $38.1 \%$ & $6.78^{\mathrm{a}}$ & $.009 *$ \\
\hline Mujer & $22.7 \%$ & $61.9 \%$ & & \\
\hline Patología Dual (sí) & $81.8 \%$ & $61.9 \%$ & $2.12^{\mathrm{a}}$ & .146 \\
\hline Politoxicomanía (sí) & $54.5 \%$ & $52.4 \%$ & $0.20^{\mathrm{a}}$ & $1^{\mathrm{b}}$ \\
\hline Tipo sustancia principal (Depresora) & $77.3 \%$ & $85.7 \%$ & $0.51^{\mathrm{a}}$ & $.698^{\mathrm{b}}$ \\
\hline Años evolución del trastorno dependencia a sustancia principal & $(22.14,10.10)$ & $(19.95,11.01)$ & $0.74^{\mathrm{c}}$ & .458 \\
\hline Ingresos Previos (sí) & $77.3 \%$ & $57.1 \%$ & $1.98^{\mathrm{a}}$ & .159 \\
\hline Despedida previa al ingreso (sí) & $22.7 \%$ & $71.4 \%$ & $10.24^{\mathrm{a}}$ & $.001 *$ \\
\hline \multicolumn{5}{|c|}{ Variables terapéuticas } \\
\hline & ABSTINENCIA & NO ABSTINENCIA & Test & $\mathrm{p}$ \\
\hline \multicolumn{5}{|l|}{ URICA Post-Grupo } \\
\hline $\begin{array}{l}\text { Precontemplacion } \\
\text { Contemplación }\end{array}$ & $(35.33,2.90)$ & $(33.60,3.13)$ & $1.49^{c}$ & .137 \\
\hline Acción & $(35.57,3.68)$ & $(33.27,3.57)$ & $1.81^{\mathrm{c}}$ & .071 \\
\hline Mantenimiento & $(32.86,3.21)$ & $(30.00,4.14)$ & $2.11^{\mathrm{c}}$ & $.035 *$ \\
\hline RTC & $(11.01,1.24)$ & $(10.16,1.53)$ & $1.85^{\mathrm{c}}$ & .064 \\
\hline \multicolumn{5}{|l|}{ CPT } \\
\hline Total & $(31.24,6.07)$ & $(27.69,5.99)$ & $1.71^{c}$ & .088 \\
\hline Terapeuta & $(15.57,3.07)$ & $(13.25,3.45)$ & $2.26^{c}$ & $.024^{*}$ \\
\hline Terapia & $(16.62,2.97)$ & $(14.44,3.42)$ & $2.04^{\mathrm{c}}$ & $.041 *$ \\
\hline
\end{tabular}

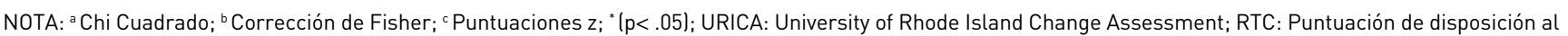
cambio; CPT: Cuestionario Percepciones de Tratamiento.

El $100 \%$ de la muestra es de nacionalidad española. En el $56.5 \%$, el alcohol es la sustancia principal, en el $21.7 \%$ la cocaína, en el $8.7 \%$ el cannabis, en el $6.5 \%$ la heroína, en el $4.3 \%$ la metadona y en el $2.2 \%$ las benzodiacepinas.

Las puntuaciones obtenidas por los participantes de la terapia en el CPT en la escala global tienen una media de $29.35(D T=6.28)$, en la subescala del terapeuta $14.52(D T=$ $3.30)$ y en la referente a la terapia $15.32(D T=3.69)$.

Considerando la sustancia principal motivo de ingreso, el $48.8 \%$ de los pacientes recaen a los dos meses. Las variables dependientes de abstinencia y retención en tratamiento posterior son asociadas. Se observa que, de los pacientes abstinentes, un $4.5 \%$ abandonan tratamiento, mientras que, de los recaídos, el porcentaje se eleva hasta el $38.1 \%$. La diferencia es estadísticamente significativa $\left(X^{2}=7.31, p=.009\right)$. Así pues, al alta de la UHD, es más probable que los que mantienen la retención al tratamiento mantengan también la abstinencia y viceversa, mientras que aquellos que no siguen en tratamiento posteriormente es más probable que tengan una recaída.

Se describen los resultados del análisis bivariante, para determinar la asociación con la abstinencia, del género (variable sociodemográfica) y de las variables clínicas y terapéuticas, tenidas en cuenta en el estudio (Tabla 2$)$. Ser mujer $\left(X^{2}\right.$ $=6.78 ; p=.009)$ y realizar una despedida de la sustancia principal previa al ingreso $\left(X^{2}=10.24 ; p=.001\right)$ se asocian de manera significativa con la recaída a los dos meses.

Respecto a las variables terapéuticas, el tener más cogniciones asociadas al estadio de mantenimiento de la abstinencia (escala de mantenimiento), logrado en la UHD, aumenta significativamente la probabilidad de mantenerla durante los dos meses posteriores $(z=2.11 ; p=.035)$. Aunque no se en- cuentran resultados significativos en la escala global del CPT, sí se hallan en ambas subescalas por separado. Puesto que las variables de satisfacción con la terapia y con el terapeuta correlacionan entre sí $(r=0.59 p<.001)$, se comparan ambos tamaños del efecto mediante la $d$ de Cohen $(d=0.68$ para satisfacción con la terapia y $d=0.71$ para el terapeuta). Se selecciona ésta última variable de las dos para la regresión logística. Se realiza el análisis multivariante para determinar si las variables que se asocian con la abstinencia, de forma bivariada a los dos meses, lo hacen de forma independiente.

$\mathrm{El}$ ser varón $(\mathrm{Wald}=6.45, p=.011, \mathrm{OR}=0.015,95 \% \mathrm{IC}=$ $0.001-0.38)$, el no realizar un atracón de la sustancia principal previa al ingreso $($ Wald $=4.61, p=.032, \mathrm{OR}=0.52,95 \%$ $\mathrm{IC}=0.004-0.77)$, mayores puntuaciones en satisfacción con el terapeuta (Wald $=4.38, p=.036, \mathrm{OR}=0.60,95 \% \mathrm{IC}=$ $0.37-0.97)$ y mayores las puntuaciones en la escala de mantenimiento del URICA (Wald $=4.47, p=.034$, OR $=0.69$, $95 \%$ IC $=0.49-0.97)$ se asocian de forma independiente con la abstinencia a los dos meses del alta.

Se realiza la regresión de Cox con el fin de determinar si los factores arriba indicados predicen un aumento del tiempo hasta la recaída (Tabla 3). Se muestra gráficamente

Tabla 3

Modelo de Cox de permanencia de la abstinencia

\begin{tabular}{lcccc}
\hline Variable & Wald & OR & IC del 95\% & p \\
\hline Género (Varón) & 7.18 & 0.20 & $0.06-0.65$ & .007 \\
Estadio Mantenimiento & 5.14 & 0.84 & $0.72-0.98$ & .023 \\
Satisfacción terapeuta & 8.10 & 0.77 & $0.64-0.92$ & .004 \\
\hline
\end{tabular}

NOTA: Género (1 = varón; 2 = mujer) 


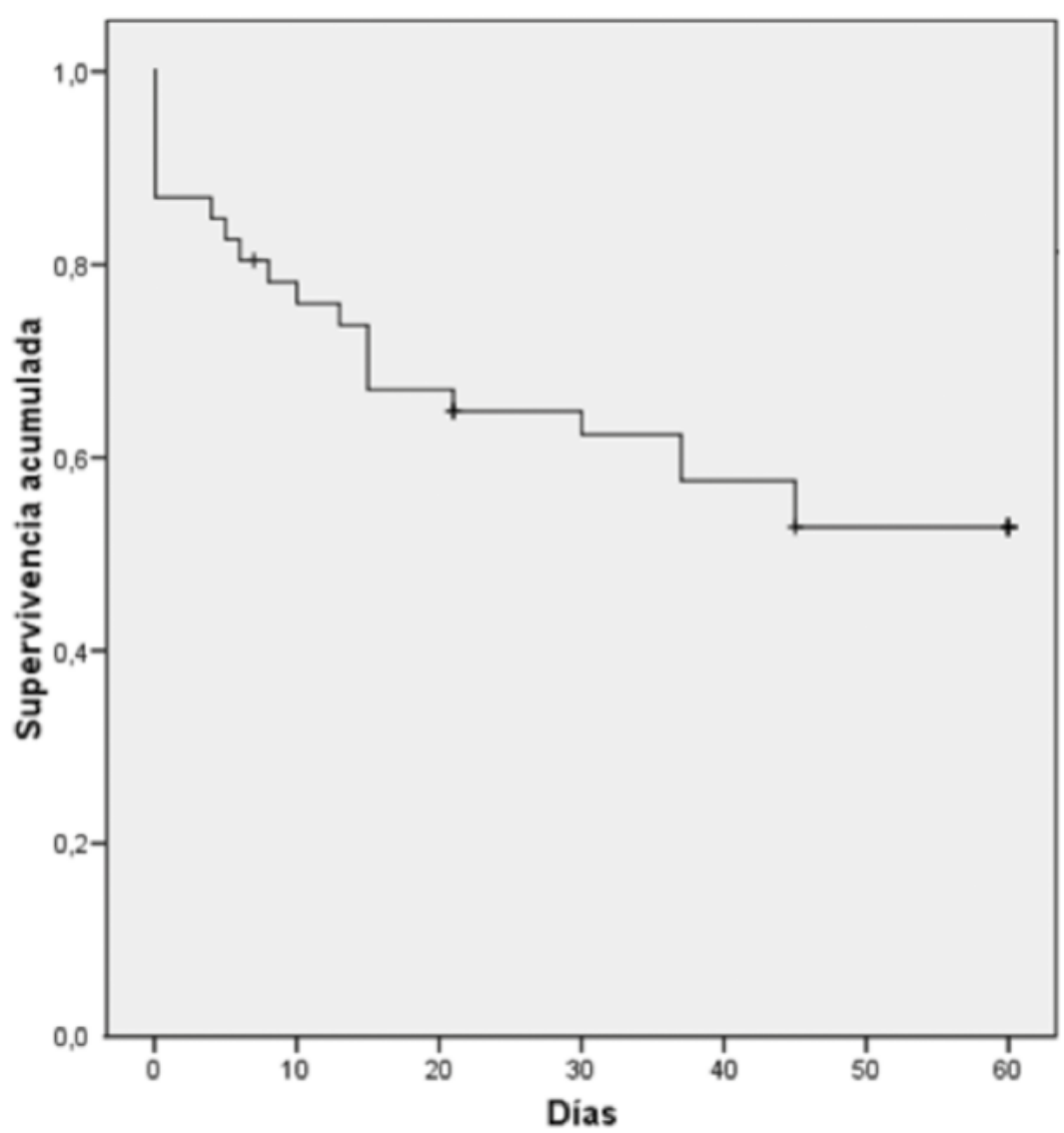

Figura 2. Estimador Kaplan-Meier de la función de Supervivencia

la función de supervivencia (Figura 2). El ser varón reduce el riesgo de la recaída en cinco veces. El tener más cogniciones y comportamientos asociados al estadio motivacional de mantenimiento, y alta satisfacción con el terapeuta grupal, también aumentan la supervivencia, controlando otras variables asociadas. Realizar una despedida de la sustancia principal, antes del ingreso, no predice un menor tiempo de supervivencia dos meses tras el alta.

\section{Discusión}

La participación en grupos terapéuticos de corte motivacional, durante el ingreso de desintoxicación, se asocia con efectos positivos sobre la probabilidad de mantener la abstinencia y la retención al tratamiento de deshabituación posterior, en un seguimiento a dos meses. Los resultados muestran que ser varón, tener mayor motivación por el cambio al alta (entendida como cogniciones del estadio de mantenimiento) y mayor satisfacción con el tratamiento psicológico durante el ingreso (con la terapia motivacional o su terapeuta), se relaciona con un mayor tiempo hasta la recaída tras la desintoxicación.

Los datos, por tanto, sugieren que tener una experiencia terapéutica satisfactoria durante el ingreso favorece la abstinencia y la retención posteriores. A este respecto existen escasos estudios en contexto de desintoxicación (Kornreich,
Dan, Fryns, Gozlan y Verbanck, 1992). En la misma línea que los resultados del presente estudio, se ha encontrado una elevada satisfacción con la terapia motivacional (Borsari y Carey's, 2000), lo que podría relacionarse con que los terapeutas de entrevista motivacional son percibidos como empáticos y con actitud de escucha (Vasilaki et al., 2006).

Por otra parte, los resultados manifiestan que una mayor disposición al cambio global (medida en la escala RTC del URICA) postratamiento, previa al alta, no se asocia con mayor probabilidad de duración de la abstinencia. Este hallazgo ya fue descrito por Diclemente, Carbonari, Zweben, Morrel y Lee (2001), quienes lo relacionaron con que la autoevaluación respecto al consumo posterior, y estadio motivacional, podría resultar optimista, influida por las restricciones y seguridad de una planta de hospitalización; por lo que dicha medición perdería capacidad predictiva en este contexto. Sin embargo, encontramos que puntuar alto en la subescala de mantenimiento se relaciona con la abstinencia, resultado que se encuentra en la misma dirección que el hallado en otro estudio anterior (Henderson, Saules y Galen, 2004). No obstante, aún no se conoce la influencia de la motivación al cambio sobre la eficacia de la entrevista motivacional y cómo mediatizaría el cambio de comportamiento (Berman et al., 2010; Dunn, Deroo y Rivara, 2001; Maisto et al., 2001; Vasilaki et al., 2006), siendo necesaria mayor investigación en este sentido. 
Uno de los objetivos principales del grupo terapéutico fue motivar la retención postalta. Este hecho podría explicar que el $73,9 \%$ de los pacientes continuaran un tratamiento de deshabituación, porcentaje que es superior al habitual en drogodependencias, pero similar a los buenos resultados obtenidos en otros programas específicos diseñados a tal efecto (Sánchez et al. 2011; Secades-Vila, García-Rodríguez, Higgins, Fernández-Hermida, y Carballo, 2008) y a los conseguidos con la introducción de grupos motivacionales en unidades de desintoxicación (Santa Ana et al., 2007). Es posible que la retención esté influida por el seguimiento telefónico semanal, aunque éste no fuera su objetivo (McKay, Lynch, Shepard y Pettinati, 2005). En cualquier caso, se trata de un resultado importante, ya que la retención es un factor predictivo de la abstinencia (Frydrych et al., 2009; Moggi, Ouimette, Moos y Finney, 1999; Secades Villa y Fernández Hermida, 2000; Vaillant, 1966); lo cual subraya de nuevo la importancia de introducir este tipo de intervenciones motivacionales, con el objetivo de mejorar el pronóstico de estos pacientes.

Continuando con los resultados obtenidos con respecto a otras variables relacionadas con la recaída, éstos indican que las mujeres presentan una mayor dificultad en el mantenimiento de la abstinencia. Este resultado coincide con lo hallado por Maehira et al., (2013). No obstante, futuros estudios deberán llevarse a cabo con el objetivo de aclarar si realmente las mujeres exhiben mayores tasas de recaída (Tuchman, 2010).

En lo concerniente a las variables clínicas examinadas, la mayoría de ellas, como es el caso de presentar un diagnóstico dual, antecedentes familiares de adicción a sustancias, la duración de la dependencia, ingresos previos, el tipo de sustancia o un patrón de policonsumo, no se asocian con una menor duración de la abstinencia. Estos resultados son congruentes con los encontrados por Loeber et al., (2009) quien halló que el tiempo hasta la primera recaída se retrasaba con independencia de la duración de la adicción al alcohol, de los ingresos previos y del género en aquellos que participaban en la terapia grupal durante el ingreso. Sin embargo, en contraste con los resultados encontrados por estos autores, en el presente estudio la variable género sí se relacionó con la duración de la abstinencia.

Aunque no predice por sí misma una menor duración del tiempo de abstinencia, el consumo a modo de despedida fue la única variable clínica que se ha asociado con la recaída, a los dos meses tras la desintoxicación. Se confirman los resultados del estudio previo (Grau-López et al., 2012), en el cual se describió esta relación a los seis meses. No obstante, parece ser que esta relación no se mantendría en seguimientos más largos, por lo menos en el caso del alcoholismo (Monrás Arnau, Gual Solé, Freixa y Lligoña, 2004).

Este estudio preliminar presenta varias limitaciones a tener en cuenta. En primer lugar, no se ha utilizado grupo control para determinar si los resultados obtenidos respecto a motivación al alta, retención en tratamiento y abstinencia posteriores se deben a la participación en el grupo terapéutico. Sin embargo, la tasa de recaída encontrada en los participantes en el grupo motivacional $(48,8 \%)$ es similar a la encontrada por Loeber et al., (2009) a los dos meses de seguimiento: un 42,4\% en comparación con el grupo control $(68,3 \%)$. Por otro lado, el disponer de un tamaño muestral pequeño y el alto porcentaje de mujeres de la muestra dificulta la generalización de los resultados a la población consumidora de sustancias que, normalmente, se distribuye con mayor porcentaje de varones (OEDT, 2012). Al tratarse de un grupo terapéutico de carácter abierto no fue posible la protocolarización de las sesiones, aunque este formato más flexible permitió la adaptación del tratamiento al contexto de la unidad con constantes ingresos y altas. Otra posible limitación es que no se usaron parámetros de laboratorio en la medición de la abstinencia, debido a la normativa de protección de datos ya que los sujetos pertenecen a distintos centros; sin embargo, se ha hallado, en otras investigaciones, que dichos parámetros no añaden mayor veracidad a la información (Babor, Steinberg, Anton y Del Boca, 2000).

El presente estudio aporta diversas contribuciones. En primer lugar, hasta donde llega nuestro conocimiento, este es el primer estudio que valora la satisfacción con grupos de corte motivacional y con el terapeuta durante el ingreso de desintoxicación y cómo se relaciona con medidas postratamiento. Además es un estudio con alta validez ecológica, al tratarse de una intervención grupal aplicable a un contexto clínico de desintoxicación hospitalaria heterogéneo en género, edad y sustancia principal.

De estos resultados se derivan diferentes líneas de investigación. En fututos trabajos, además de medir el estadio motivacional (DiClemente, Schlundt y Gemmell, 2004), se deberían considerar aspectos cognitivos más implícitos, por ejemplo, creencias nucleares relacionadas con la adicción (Martínez González y Verdejo García, 2012). Por otra parte, los componentes más básicos de la satisfacción, con la terapia o con el terapeuta, y su influencia sobre la eficacia del tratamiento psicológico en drogodependencias, deben ser investigados para poder así mejorar dichas intervenciones.

Por último, estos datos poseen relevantes implicaciones clínicas, ya que demuestran la importancia de ofrecer tratamiento psicosocial añadido al tratamiento farmacológico desde las primeras fases de la intervención con adictos con el objetivo de mejorar su eficacia (Berner et al., 2008; Loeber et al., 2009; Stetter y Mann, 1997).

Se concluye, por tanto, que los aspectos relacionados con la intervención grupal de corte motivacional, como son la satisfacción con el terapeuta, con la terapia, y el presentar cogniciones asociadas al mantenimiento de la abstinencia en el momento del alta, operan como factores protectores, ya que se asocian con mayor duración de la abstinencia tras el ingreso. 


\section{Reconocimientos}

Queremos agradecer al Dr. Joan Colom (Subdirección General de Drogodependencias. Departamento de Salud. Generalitat de Catalunya) por su soporte a la Unidad Hospitalaria de Desintoxicación Vall Hebron y al Instituto Carlos III : FIS PI13/1911 por el estudio de las drogodependencias. También al equipo de enfermería, a las psicólogas que participaron en la evaluación de pacientes Nuria Tarifa, Sonia Fuentes, Yasmina Pallarés, Alba Saumel, y al Dr. Egido. Queremos agradecer especialmente a los psicólogos internos residentes por su trabajo terapéutico, seguimiento de los pacientes y colaboración en el presente artículo (Francisco Villar, Clara Vila, Maria José Fernández, Pilar Giráldez, Raquel Vidal, Cristina Valls, Marta Perea, Alba González, Anna Beneria y Patricia Padilla).

\section{Conflicto de intereses}

No existen conflictos de intereses.

\section{Referencias}

Arias, F., Szerman, N., Vega, P., Mesias, B., Basurte, I., Morant, C.,... Babín, F. (2013). Estudio Madrid sobre prevalencia y características de los pacientes con patología dual en tratamiento en las redes de salud mental y de atención al drogodependiente. Adicciones, 25, 118-127.

Babor, T. F., Steinberg, K., Anton, R., y Del Boca, F. (2000). Talk is cheap: measuring drinking outcomes in clinical trials. Journal of Studies on Alcohol, 61, 55-63.

Berman, A. H., Forsberg, L., Durbeej, N., Kallmen, H. y Hermansson, U. (2010). Single-session motivational interviewing for drug detoxification inpatients: effects on self-efficacy, stages of change and substance use. Substance Use E Misuse, 45, 384-402. doi: $10.3109 / 10826080903452488$

Berner, M., Günzler, C., Frick, K., Kriston, L., Loessl, B., Brück, R.,... Mann, K. (2008). Finding the ideal place for a psychotherapeutic intervention in a stepped care approach-a brief overview of the literature and preliminary results from the Project PREDICT. International Journal of Methods in Psychiatric Research, 17 (Supl. 1), 6064. doi: 10.1002/mpr.250

Borsari, B. y Carey, K. B. (2000). Effects of a brief motivational intervention with college student drinkers. Journal of Consulting and Clinical Psychology, 68, 728-733.

Chutuape, M. A., Katz, E. C. y Stitzer, M. L. (2001). Methods for enhancing transition of substance dependent patients from inpatient to outpatient treatment. Drug and Alcohol Dependence, 61, 137-143.

DiClemente. C. C., Carbonari, J., Zweben, A, Morrel, T. y Lee, R. L. (2001). Motivation Hypotheis Causal chain Analysis. En R. Longabaugh y P. W. Wirtz (Eds.), Project MATCH Hipotheses: Results and Causal Chain Analyses.
Project MATCH Monograph Series, Volume 8. (pp. 239-259). Rockville, MD: National Institute on Alcohol Abuse and Alcoholism.

DiClemente, C. C., Schlundt, D. y Gemmell, L. (2004). Readiness and stages of change in addiction treatment. The American Journal on Addictions, 13, 103-119.

Driessen, M., Veltrup, C., Junghanns, K., Przywara, A. y Dilling, H. (1999). Cost-efficacy analysis of clinically evaluated therapeutic programs. An expanded withdrawal therapy in alcohol dependence. Der Nervenarzt, 70, 463470.

Dunn, C., Deroo, L. y Rivara, F. P. (2001). The use of brief interventions adapted from motivational interviewing across behavioral domains: a systematic review. Addiction, 96, 1725-1742. doi: 10.1080/09652140120089481

First, M. B., Gibbon, M., Spitzer, R. L., Williams, J. B. W. y Benjamin, L. S. (1999). Entrevista clínica estructurada para los trastornos de la personalidad del Eje II del DSM-IV (SCIDII). Barcelona: Masson.

First, M. B., Spitzer, R. L., Gibbon, M. y Williams, J. B. W. (1999). Entrevista clínica estructurada para los trastornos del Eje I del DSM-IV, versión clínica (SCID-I-VC). Barcelona: Masson.

Frydrych, L. M., Greene, B. J., Blondell, R. D. y Purdy, C. H. (2009). Self-help program components and linkage to aftercare following inpatient detoxification. Journal of Addictive Diseases, 28, 21-27. doi: 10.1080/10550880802544799

García Rodríguez, O., Secades Villa, R., Fernández Hermida, J. R., Carballo Crespo, J. L., Errasti Pérez, J. M. y Al-Halabí Díaz, S. (2005). Comparación de pacientes cocainómanos y heroinómanos en el EuropASI. Adicciones, 17, 33-42.

Goeb, J. L., Coste, J., Bigot, T. y Ferrand, I. (2000). Prospective study of favorable factors in follow-up of drug addicted patients-apropos of 257 patients of the Cassini Center in Paris. L'Encéphale, 26, 11-20.

Grau-López, L., Roncero, C., Daigre, C., Gonzalvo, B., Bachiller, D., Rodriguez-Cintas, L.,... Casas, M. (2012). Factores de riesgo de recaída en pacientes drogodependientes tras desintoxicación hospitalaria. Adicciones, 24, 115-122.

Henderson, M. J., Saules, K. K. y Galen, L. W. (2004). The predictive validity of the University of Rhode Island Change Assessment Questionnaire in a heroin-addicted polysubstance abuse sample. Psychology of Addictive Behaviors, 18, 106-112. doi: 10.1037/0893-164X.18.2.106

Ino, A., Saka, Y., Yamashiro, K., Cho, T. y Torituka, M. (2006). Alcohol dependence syndrome and Before-Discharge Intervention Method (BDIM)-No. 5. Patients' evaluation of BDIM by questionnaire survey. Japanese Journal of Alcohol Studies Ẽ Drug Dependence, 41, 358-367.

John, U., Veltrup, C., Driessen, M., Wetterling, T. y Dilling, H. (2003). Motivational intervention: an individ- 
ual counselling vs a group treatment approach for alcohol-dependent in-patients. Alcohol and Alcoholism, 38, 263-269.

Kornreich, C., Dan, B., Fryns, A., Gozlan, S. y Verbanck, P. (1992). Cognitive-behavioral approach to alcoholism: preliminary report of the realization of a therapeutic group. Acta Psychiatrica Belgica, 92, 279-293.

Loeber, S., Kiefer, F., Wagner, F., Mann, K. y Croissant, B. (2009). Treatment outcome after inpatient alcohol withdrawal: impact of motivational interventions: a comparative study. Der Nervenarzt, 80, 1085-1092. doi: 10.1007/ s00115-009-2724-2

Maehira, Y., Chowdhury E.I., Reza, M., Drahozal, R., Gayen, T.K., Masud, I.,... Azim, T. (2013). Factors associated with relapse into drug use among male and female attendees of a three-month drug detoxification-rehabilitation programme in Dhaka, Bangladesh: a prospective cohort study. Harm Reduction Journal, 10, 1-14. doi: 10.1186/1477-7517-10-14

Maisto, S. A., Conigliaro, J., McNeil, M., Kraemer, K., Conigliaro, R. L. y Kelley, M. E. (2001). Effects of two types of brief intervention and readiness to change on alcohol use in hazardous drinkers. Journal of Studies on Alcohol, 62, 605-614.

Mandersen, J., Nizzoli, U., Corbelli, C., Margaron, H., Torres, M.A.,... Gossop, M. (2001). Fiabilidad del "Maudsley addiction profile" (MAP, ERIR-Versión) y del "Treatment perceptions questionnaire (TPQ) en Italia, España y Portugal para la evaluación de tratamientos. Adicciones, 13, 217-227.

Márquez-Arrico, J. E. y Adán, A. (2013). Patología dual y rasgos de personalidad: situación actual y líneas futuras de trabajo. Adicciones, 25, 195-202.

Marsden, J., Bacchus, L., Stewart, D., Griffiths, P., Clarke, K. Gossop, M. y Strang, J. (1998). The Treatment Perceptions Questionnaire (TPQ): A brief questionnaire for assessing service satisfaction. Manuscrito no publicado. Londres: National Addiction Centre.

Martínez González, J. M., y Verdejo García, A. (2012). Evolución de las creencias nucleares relacionadas con la adicción en drogodependientes con y sin trastornos de personalidad. Adicciones, 24, 229-238.

McConnaughy, E. A., DiClemente, C. C., Prochaska, J. O., y Velicer, W. F. (1989). Stages of change in psychotherapy: A follow-up report. Psychotherapy, 26, 494-503. doi: $10.1037 / \mathrm{h} 0085468$

McConnaughy, E. A., Prochaska, J. O. y Velicer, W. F. (1983). Stages of change in psychotherapy: Measurement and sample profiles. Psychotherapy: Theory, Research, and Practice, 20, 368-375. doi: 10.1037/h0090198

McKay, J. R., Lynch, K. G., Shepard, D. S. y Pettinati, H. M. (2005). The effectiveness of telephone-based continuing care for alcohol and cocaine dependence: 24-month outcomes. Archives of General Psychiatry, 62, 199-207. doi: 10.1001/archpsyc.62.2.199

Miller, W. R. y Rollnick, S. (2002). Motivational Interviewing: Preparing people for change (2nd ed.). Nueva York: Guilford Press.

Moggi, F., Ouimette, P. C., Moos, R. H. y Finney, J. W. (1999). Dual diagnosis patients in substance abuse treatment: relationship of general coping and substance-specific coping to 1-year outcomes. Addiction, 94, 1805-1816. doi: 10.1046/j.1360-0443.1999.941218056.x

Monrás Arnau, M., Gual Solé, A., Freixa, N. y Lligoña, A. (2004). El último consumo de alcohol previo al ingreso de desintoxicación como predictor a largo plazo de la abstinencia. Adicciones, 16, 117-122.

Myrick, H., Anton, R. y Kasser. C. (2003). Management of intoxication and withdrawal; general principles. En: A.W. Graham, T. K. Schultz, M. F. Mayo-Smith, R. K. Ries y B. B. Wilford (Eds.), Principles of addiction medicine (3rd ed.) (pp. 611-618). Chevy Chase, MD: American Society of Addiction Medicine.

National Institute on Drug Abuse. (2010). Principios de tratamientos para la drogadicción: Una guía basada en las investigaciones (2nd ed.). Washington, DC: Institutos Nacionales de la Salud. Recuperado de http:/ / www.drugabuse.gov/sites/default/files/podatsp.pdf

Observatorio Español de Drogas y Toxicomanías (OEDT) para el Plan Nacional sobre Drogas (2012). Informe Nacional 201 al OEDT por el punto focal nacional Retoix Madrid: Ministerio de Sanidad, Servicios Sociales e Igualdad. Recuperado de http://www.pnsd.msc.es/Categoria2/ publica/pdf/Informe_Nacional_REITOX2012.pdf

O'Farrell, T. J., Murphy, M., Alter, J. y Fals-Stewart, W. (2008). Brief family treatment intervention to promote aftercare among substance abusing patients in inpatient detoxification: Transferring a research intervention to clinical practice. Addictive Behaviors 33, 464-471.

Prochaska, J. O. y DiClemente, C. C. (1982). Trans-theoretical therapy - toward a more integrative model of change. Psychotherapy: Theory, Research and Practice 19, 276-288.

Rojas, M. J. y Espinoza, L. S. (2008). Consejo psicológico y psicoterapia motivacional en drogodependencias: evaluación de las intervenciones del programa "Lugar de Escucha”. Adicciones, 20, 185-196.

Sánchez, L., Díaz-Morán, S., Grau-López, L., Moreno, A., Eiroa-Orosa, F. J., Roncero, C.,... Casas, M. (2011). Tratamiento ambulatorio grupal para dependientes de cocaína combinando terapia cognitiva conductual y entrevista motivacional. Psicothema, 23, 107-113.

Santa Ana, E. J., Wulfert, E. y Nietert, P. J. (2007). Efficacy of group motivational interviewing (GMI) for psychiatric inpatients with chemical dependence. Journal of Consulting and Clinical Psychology, 75, 816-822. doi: 10.1037/0022-006X.75.5.816 
Schellekens, A. F., de Jong, C. A., Buitelaar, J. K. y Verkes, R. J. (2015). Co-morbid anxiety disorders predict early relapse after inpatient alcohol treatment. European Psychiatry, 30, 128-136. doi: 0.1016/j.eurpsy.2013.08.006

Schilling, R. F., El-Bassel, N., Finch, J. B., Roman, R. J. y Hanson, M. (2002). Motivational Interviewing to encourage self-help participation following alcohol detoxification. Research on Social Work Practice, 12, 711-730. doi: 10.1177/104973102237469

Secades Villa, R. y Fernández Hermida, J. R. (2000). Cómo mejorar las tasas de retención en los tratamientos de drogodependencias. Adicciones, 12, 353-63.

Secades-Villa, R., García-Rodríguez, O., Higgins, S. T., Fernández-Hermida, J. R. y Carballo, J. L. (2008). Community reinforcement approach plus vouchers for cocaine dependence in a community setting in Spain: six month outcomes. Journal of Substance Abuse Treatment, 34, 202207.

Shaw, G. K., Waller, S., Latham, C. J., Dunn, G. y Thomson, A. D. (1998). The detoxification experience of alcoholic in-patients and predictors of outcome. Alcohol and Alcoholism (Oxford, Oxfordshire), 33, 291-303.

Stetter, F. y Mann, K. (1997). Dependency outcome of alcohol-dependent patients after inpatient detoxification and motivation treatment. Der Nervenarzt, 68, 574-581.
Stetter, F., Zahres, S., Batra, A. y Mann, K. (1995). Results of integrated inpatient detoxification and motivation treatment of alcohol dependent patients. Psychiatrische Praxis, 22, 189-192.

Tuchman, E. (2010). Women and addiction: the importance of gender issues in substance abuse research. Journal of Addictive Diseases, 29, 127-138. doi: $10.1080 / 10550881003684582$

Vaillant, G. E. (1966). A twelve-year follow-up of new york narcotic addicts: I. The relation of treatment to outcome. American Journal of Psychiatry, 122, 727-737.

Vasilaki, E. I., Hosier, S. G. y Cox, W. M. (2006). The efficacy of motivational interviewing as a brief intervention for excessive drinking: a meta-analytic review. Alcohol and Alcoholism, 41, 328-335. doi: 10.1093/alcalc/ag1016

Volpicelli, J. R., Rhines, K. C., Rhines, J. S., Volpicelli, L. A., Alterman, A. I. y O'Brien, C.P. (1997). Naltrexone and alcohol dependence. Role of subject compliance. Archives of General Psychiatry, 54, 737-742.

Wagner, C. C. y Ingersoll, K. S., (2012). Motivational Interviewing in groups. Nueva York: Guilford Press.

Wierzbicki, M. y Pekarik, G. (1993). A meta-analysis of psychotherapy dropout. Professional Psychology: Research and Practice, 24, 190-195. doi: 10.1037//0735-7028.24.2.190 